\title{
Validity of intraoral approach in management of infraorbital rim fracture
}

\author{
Original \\ Article \\ Khaled I. Barakat ${ }^{a}$, Mohamed S. Abdelmonem ${ }^{b}$ and Youssef A. ElMansy ${ }^{c}$ \\ Department of Oral and Maxillofacial Surgery, ${ }^{a}$ Faculty of Dentistry, Minia \\ University, Minia, ${ }^{c}$ Military Academy, Military Hospitals, ${ }^{b}$ Department of Oral and \\ Maxillofacial, Faculty of Dentistry, Mina University, Mina, Egypt
}

\begin{abstract}
Objectives: Lower lid approaches are vulnerable to several complications as they disrupt the complex anatomy of the lower palpebrae. Currently intra-oral approaches are replacing those standard approaches, but unfortunately, they suffer limitations in their accessibility. Thus, we aim to evaluate the intraoral approach in management of infraorbital rim fracture considering exposure and complications.

Patients and Methods: Twelve patients suffering displaced infraorbital rim fracture. All patients diagnosed clinically and radiographically, then treated by open reduction and internal fixation (ORIF). Examined parameters were exposure extent and complications (edema, and patient satisfaction).

Conclusion: Intraoral approach is a valid technique for reduction and fixation of infraorbital rim. It permits enough exposure with minimal complications and maximal patient satisfaction.
\end{abstract}

Key Words: Infraorbital rim, Intraoral approach, Maxillofacial trauma, Orbital fracture, Zygomaticomaxillary complex.

Received: 20 October 2020, Accepted: 28 April 2021.

Corresponding Author: Khaled Barakat, Department of Oral and Maxillofacial Surgery, Faculty of Dentistry, Minia University, Minia, Egypt, Tel.: +20222677077, Mobile: +201222324596, E-mail: send2kbarakat@yahoo.com.

ISSN: 2090-097X, October 2020, Vol. 11, No. 4

\section{INTRODUCTION}

Fracture of infraorbital rim either isolated or associated with ZMC or orbital fractures is one of the most challenging types of trauma in maxillofacial surgery.

The zygoma is geometrically unique because it articulates with four bones: frontal, temporal, maxilla and sphenoid. Zygomatic fractures are typically described as being tetrapod ${ }^{[1]}$. Fracture of the zygomatic bone represents $42 \%$ of facial fractures and represents $64 \%$ of all middle third fractures due to the anatomical position of the zygomatic bone.

Moreover, orbital fractures represent one of the most common conditions encountered today in our mechanized life ${ }^{[2]}$.

Since 1998, ORIF became the standard management for displaced fractures. It provides stable reconstruction, promotes bone healing, shortens treatment time and allows immediate jaw mobilization ${ }^{[3]}$.

Several skin approaches have been devised to access this area. However, these approaches disrupt the delicate and complex anatomy of the lower eye lid. Orbicularis muscle, tarsus, septum, and other vital structures are difficult to regain their normal anatomic position and function.

Subciliary, subtarsal, and transconjunctival approaches provide wide exposure to the orbital floor and infraorbital rim fractures ${ }^{[4]}$, but they involve several complications.
Hypertrophic scar formation, scleral show, mild lid edema, keratoconjunctivitis, epiphora, ectropion, entropion, lagophthalmos, and nasolacrimal injury ${ }^{[5]}$. Thus, these techniques should be limited to cases of orbital extended walls fixation.

The simplest method of repair should be chosen whenever as it is effective as more invasive one following the four main principles for surgical repair of the maxillofacial fracture: enough exposure, adequate reduction, stable fixation, and least complications ${ }^{[6]}$.

In order to overcome this predicament, some authors devised intraoral approach for intraoral reduction and fixation. They concluded that, this technique suffering limitation in accessibility of exposure.

The aim of current study was to evaluate the validity of intraoral approach in management of infraorbital rim fracture considering exposure and complications.

\section{MATERIALS AND METHODS}

\section{Study Design:}

This study was conducted at the Oral and maxillofacial surgery Department, Faculty of Dentistry, Minia University, and Egyptian Military Hospitals. The protocol for the study was approved by the Ethical Committee of the faculty and informed consent was obtained from the participating patients. 
Twelve patients ( 8 males, 4 females, age range 18 to 49 years [average age 37.3 years]) suffering from infraorbital rim fracture were managed by ORIF via intraoral approach.

\section{Inclusion Criteria:}

1. Infraorbital rim fracture (unilateral or bilateral) diagnosed clinically and confirmed by CT.

2. Age ranging from $18-50$ year.

\section{Exclusion Criteria:}

1. Medically compromised patients (metabolic bone disease, patients with history of radiotherapy and uncontrolled diabetic patient).

2. Patients with orbital floor fracture when access to the orbital floor is necessary.

3. Patients with soft tissue laceration in the zygomatic region that allows infraorbital rim exposure.

4. Non-displaced ZMC fractures that conservative treatment is satisfactory.

5. Patients with comminuted fracture orbital rim, with more than $5 \mathrm{~mm}$ of displacement of fractured segments.

6. Preoperative infection.

\section{Preoperative Examination:}

A detailed history and clinical examination records were reviewed for age, sex, cause of injury, diagnosis, side of injury, associated facial fractures, abnormal eye signs, mouth opening and cosmetic manifestations preoperatively. Facial bones inspected and palpated for asymmetry caused by displaced fragments of the facial skeleton and for areas of edema, ecchymosis, and lacerations.

\section{MSCT:}

All patients were subjected for multi-slice Computed tomography (MSCT) axial, coronal, sagittal slice thickness $1 \mathrm{~mm}$ and 3D reconstruction images (Figure 1).
All patients were started on Pre-Operative intravenous (IV) antibiotics (Ceftriaxone 1gm. IV every 12 hours, Metronidazole 500mg. IV every 8 hours) and analgesic (diclofenac $75 \mathrm{mg}$. IV every 8 hours) in $100 \mathrm{ml}$-isoosmotic dextrose for 3 - 4 days. Most of the patients had periorbital edema and were operated five to seven days after admission.

\section{Surgical Procedures:}

The surgical procedure was performed under general anesthesia with nasotracheal intubation (NTI). A horizontal maxillary vestibular incision was performed from the lateral incisor to the first molar in the same side to access the infraorbital rim (Figure 2), the mucoperiostium flap was elevated exposing the anterior wall of the maxilla, lateral wall of the nose.

Apart of the masseter and zygomaticus major muscles were detached from the inferior border of the zygoma and the zygomatic process of the maxilla exposing the zygomatic body and a part of the zygomatic arch.

The infraorbital nerve was dissected to tunnelize the infraorbital nerve above the infraorbital foramen and the piriform aperture to obtain greater motion of the flap (Figure 3).

After exposure, the reduction of the fracture was performed via held the infraorbital rim in anatomical position. Orbital mini-plate (Module $2.0 \mathrm{~mm}$, Color: yellow) at least 6 holes, two fixation point in the same part of fracture for stability, the mini-plates was adapted over infraorbital rim (STEMA Medizintechnik, 1.0 thickness).

Drilling is done semi perpendicular and screw fixation was then performed by two mini-screws at each side of each fracture line at least (Figure 4). After fracture repair, the soft tissue closure was done by sutures 3 - 0 resorbable suture.

Clinical exposure was determined by the exposed holes of the standard orbital mini-plates 6 - 10 holes that placed and adapted on infraorbital rim when retract medial and lateral together. Post-operative evaluation: Post-operative MSCT were performed to assess accuracy of reduction and fixation.

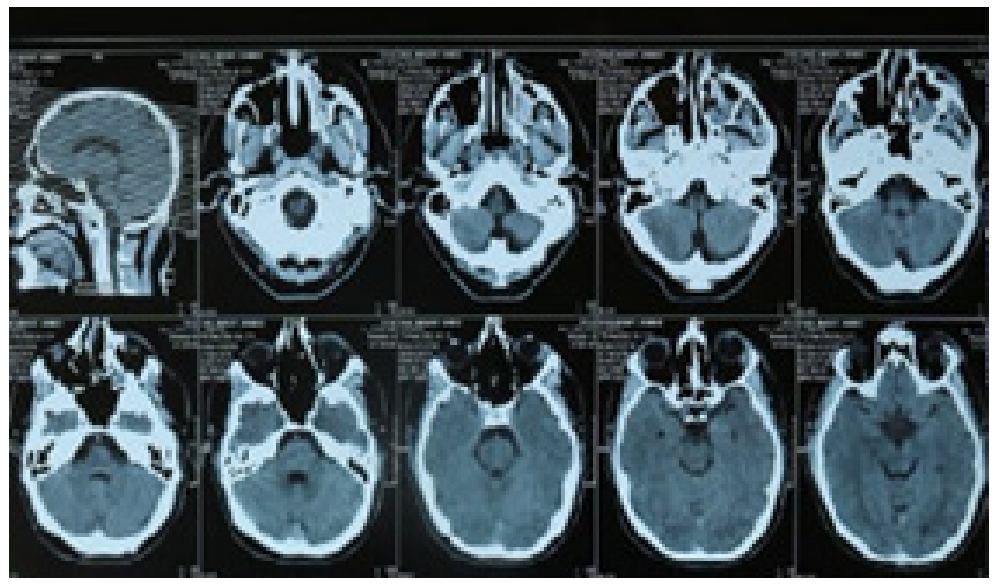

Figure 1: (MSCT) axial cuts for facial bones. 


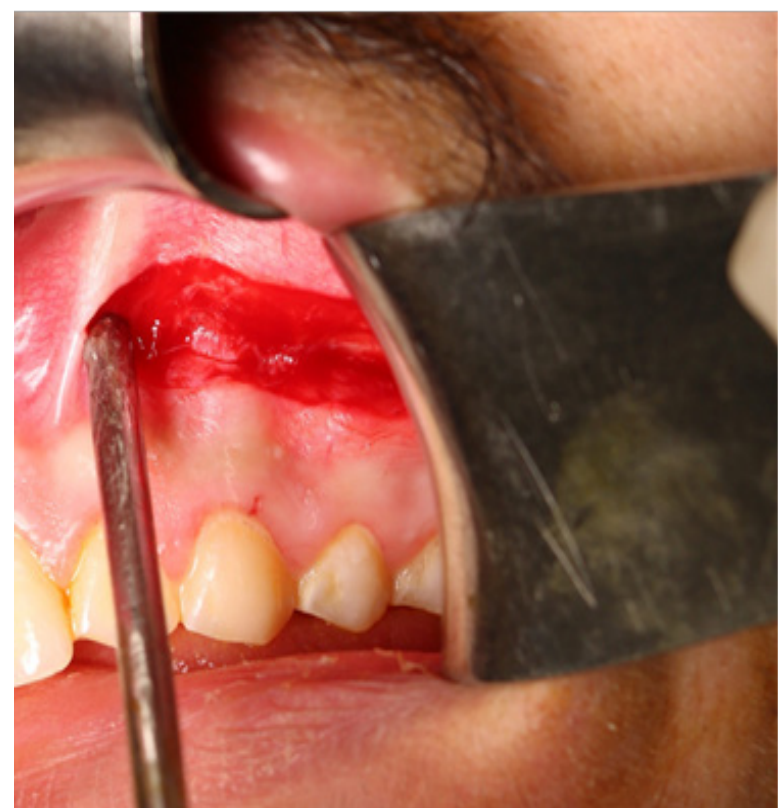

Figure 2: Incision from lateral to first molar.

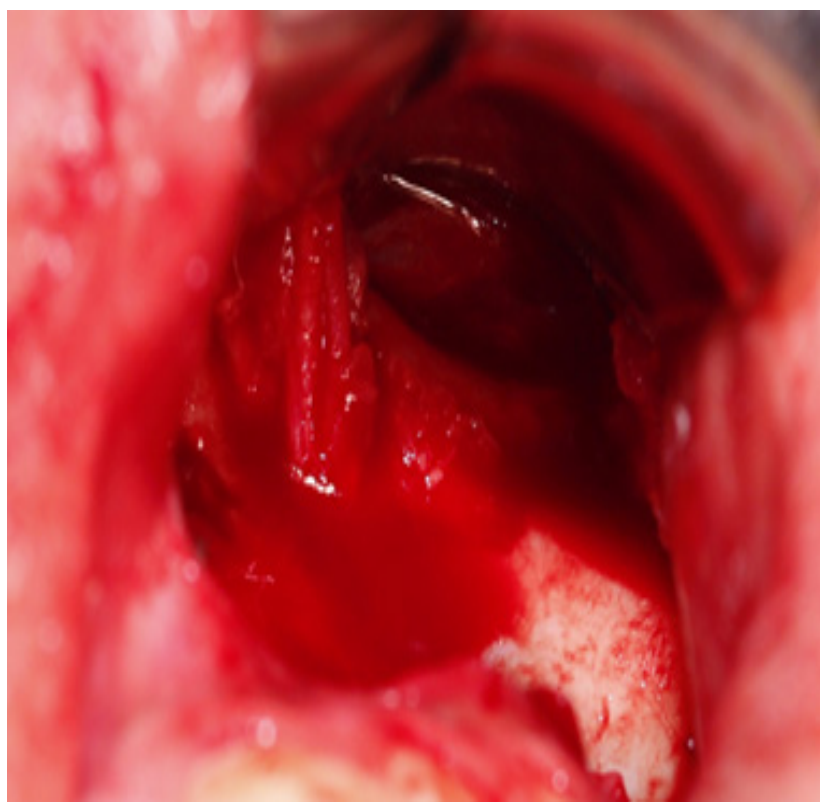

Figure 3: Tunneling of infraorbital nerve.

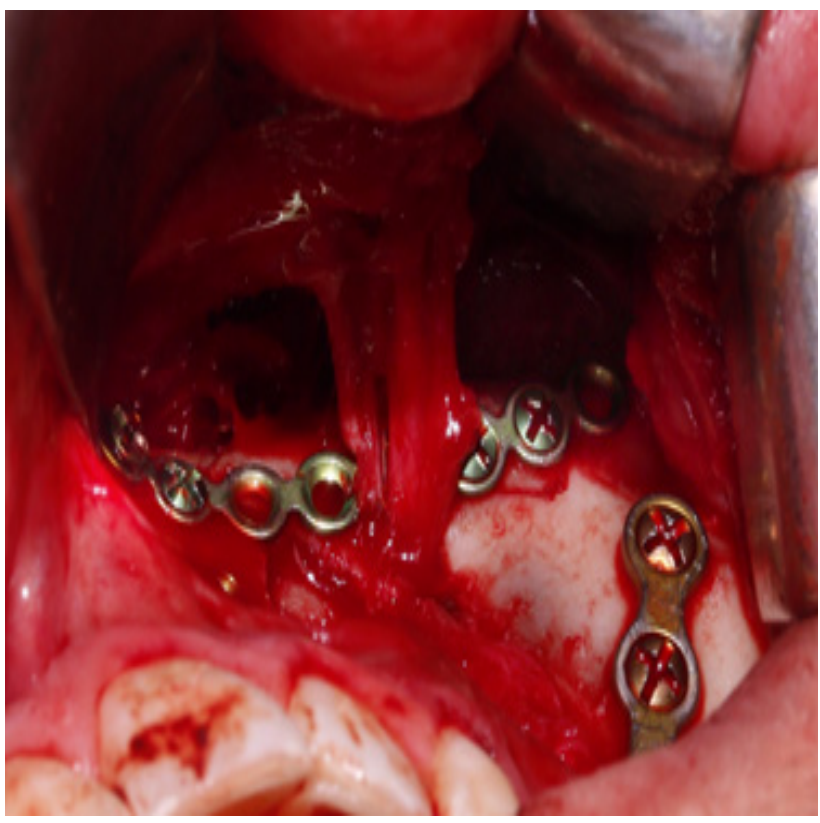

Figure 4: Fixation with orbital mini-plates.

\section{Clinical evaluation was done using:}

1. Edema evaluated by modification laskin ${ }^{[7]}$ method, which consist of 3-line measurement by 4 fixed points on the surgical side of the face. The fixed points used are $\mathrm{A}$; the most posterior point on the tragus, $\mathrm{B}$; lateral canthus of the eye, $\mathrm{C}$; the most lateral point on the angle of the mouth, and D; most inferior point on the angle of the mandible.

The 3 lines are $\mathrm{AB}, \mathrm{AC}$, and $\mathrm{BD}^{[8]}$ (Figure 5).

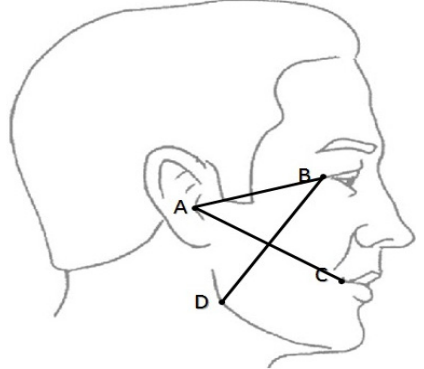

Figure 5: Modification laskin method, 4 fixed points, 3-line measurements on the surgical side of the face. 
Points are marked with a demographic pencil and lines determined by suture 3 - 0 which measured by digital caliper. These measurements are distributed at the following times: post trauma in emergency room, after one day of surgery, after one week and after one month which was compared with other side of face. The sum of the three lines measures edema index, mean and average was calculated and recorded.

2. Subjective satisfaction questionnaire which consists of 7 questions that assess: Nerve numbness (question 1, 2, 3), facial asymmetry (question 4, 5), pain (question 6) and overall satisfaction (question 7). The assessment was performed according the result of Q7 either satisfied or not after one month following the surgery (Table 1).

\section{Ethical Considerations:}

All reasonable steps to protect the security of the personal information and privacy of the patient protected health had been taken. All data had been kept confidential and the Faculty of Research Ethics Committee had reviewed the proposal. All patients had been informed in detail about the nature of the investigation and the purpose of the study; all of them had agreed and signed on an informed consent.

Table 1: Subjective questionnaire:

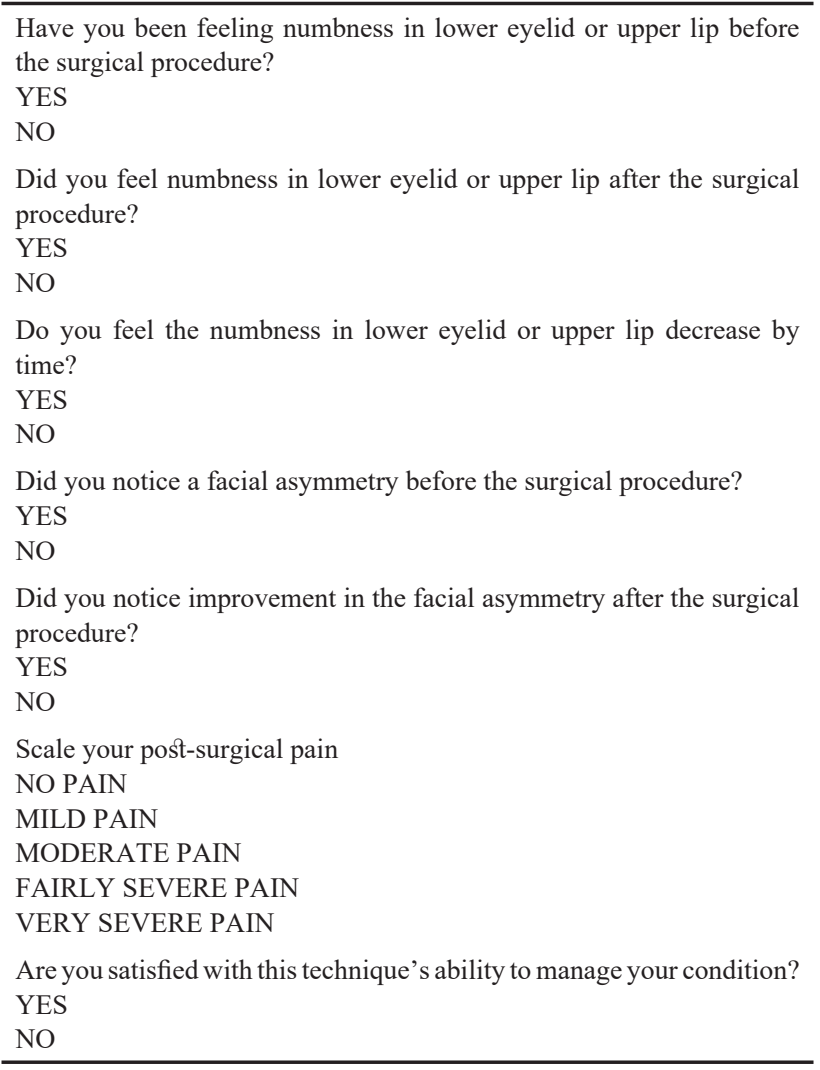

\section{Postoperative instructions:}

Keeping the patient's head in an upright position both preoperatively and postoperatively to improve periorbital edema and pain, apply ice packs for the first 24 hours (may be effective in the short term to minimize edema) and soft diet should be instructed as tolerated until there has been adequate healing of the maxillary vestibular incision.

\section{Postoperative mediation:}

Continued the same preoperative regimen with Steroids (4 mg dexamethasone twice a day) for 7 days and regular perioral for 7 days and oral wound care has to include disinfectant mouth rinse, lip care, etc...

\section{Follow-up:}

After 1st day to record edema, oral hygiene measure instruction and obtain $\mathrm{CT}$ and $3 \mathrm{D}$ reconstruction radiograph postoperative, after one week to assess the edema, after one month to assess edema and fulfill the subjective questionnaire that recorded the result.

\section{Statistical analysis:}

Recorded data that included:

1. Exposure of infraorbital rim by using the standard orbital mini-plates.

2. Edema by using 3-line measurements.

3. Satisfaction of patient using subjective questionnaire were analyzed using the statistical package for social sciences, version 20.0 (SPSS Inc., Chicago, Illinois, USA). Quantitative data were expressed as mean \pm standard deviation (SD). Qualitative data were expressed as frequency and percentage.

- Paired sample t-test of significance was used when comparing between related sample.

- $P$-value $<0.05$ was considered significant.

\section{RESULTS}

The mean age of the patients was $29.58 \pm 9.76$ years (range 18 - 49 years). There were 4 (33.3\%) female and $8(66.7 \%)$ males. Distribution of cases according to their etiology of fracture were: the ASSULT 10 (83.3\%), RTA $1(8.3 \%)$ and FALL $1(8.3 \%)$ of etiology of fracture.

The mean percentage holes exposed of standard orbital mini-plates exposed was 8 holes (Table 2). 
Table 2: Descriptive of cases according to the exposed holes of standard mini orbital plates:

$\begin{array}{lll}\text { Exposure } & \text { Range } & \text { Mean } \pm \text { SD } \\ \text { Plate holes } & 6-10 & 8.00 \pm 1.48 \\ \text { Exposed } & 6-8 & 7.08 \pm 0.79 \\ \text { Percentage } & 80-100 & 89.79 \pm 8.15\end{array}$

$(\mathrm{n}=12)$

Edema index assessment by 3-line measurements, revealed statistically significant difference between post trauma in emergency room, after 1 day and after 1 month (Table 3).

Table 3: 3-lines measurement assessment of edema index:

\begin{tabular}{lccccl}
\hline \multirow{1}{*}{ Period } & \multicolumn{2}{c}{ Edema } & \multicolumn{3}{c}{ Paired Sample t-test } \\
\cline { 2 - 6 } & Range & Mean \pm SD & $\begin{array}{c}\text { Mean } \\
\text { Diff. }\end{array}$ & t-test & p-value \\
\hline $\begin{array}{l}\text { Post trauma } \\
\text { in emergency }\end{array}$ & $32-37$ & $34.83 \pm 1.59$ & & & \\
room & & & & & \\
After 1 day & $36-40$ & $38.08 \pm 1.24$ & -3.250 & -8.298 & $<0.001 * *$ \\
After 1 week & $32-37$ & $34.92 \pm 1.38$ & -0.083 & -0.178 & 0.862 \\
After 1 month & $30-35$ & $32.00 \pm 1.65$ & 2.833 & 4.145 & $0.002 *$ \\
\hline$p$-value $<0.05 \mathrm{NS} ; * p$-value $<0.05 \mathrm{~S} ; * * p$-value $<0.001 \mathrm{HS}$.
\end{tabular}

In comparison between both sides after one month, there were a statistically significant difference between the affected and unaffected side (Table 4).

Table 4: Edema index assessment comparison between both sides after one month:

\begin{tabular}{|c|c|c|c|c|c|}
\hline \multirow[b]{2}{*}{ Period } & \multicolumn{2}{|c|}{ Edema } & \multicolumn{3}{|c|}{ Paired Sample t-test } \\
\hline & Range & Mean \pm SD & $\begin{array}{l}\text { Mean } \\
\text { Diff. }\end{array}$ & t-test & p-value \\
\hline $\begin{array}{ll}\text { After } 1 \\
\text { month }\end{array}$ & $30-35$ & $32.00 \pm 1.65$ & & & \\
\hline $\begin{array}{l}\mathrm{O} t \mathrm{~h} \text { e } \mathrm{r} \\
\text { side of } \\
\text { face }\end{array}$ & $29-35$ & $31.67 \pm 1.83$ & 0.333 & 1.345 & 0.139 \\
\hline
\end{tabular}

According to the result of Q7 of subjective satisfaction questionnaire of surgery: the satisfied patients $11(91.7 \%)$ and unsatisfied $1(8.3 \%)$ (Figure 6).

\section{DISCUSSION}

Various approaches have been used and described in literatures to visualize and access the infraorbital rim and the orbital floor over the years.

According to Padmanavam et $a l^{[9]}$ Upper Buccal Sulcus approach was used for reduction of the fracture satisfactorily, with the advantages of no skin scar, closer and more precise application of force by the operator, placement of bone plates at the buttress possible through the same incision, minimal bleeding, simplified antral bone harvest if required, and simple mucosal closure.

In 2012 Abrahao, $\mathrm{C}$ used this technique and concluded that intraoral technique allows an approach to the zygomaticomaxillary buttress and infraorbital rim with only 1 incision, optimizes surgical time, decreases complication rates, and avoids periorbital scarring in the patient ${ }^{[10]}$, Shikara $\mathrm{MA}^{[11]}$ also reported that the buccal approach provided satisfactory outcomes with no additional morbidity or injury to the eye structures, as well as resolution of the preoperative numbness. There were no other major complications reported for the sublabial approach.

On the other hand, Yoneharaet al reported that inferior orbital rim fixation through a subciliary incision or transconjunctival lower lid incision can cause complications such as bleeding, hematoma formation, infection, scarring, contracture, and ectropion of the lower eyelid, however ${ }^{[12]}$. According to our result, we used standard mini orbital plate as a reference to assess the exposure extent. The 6-hole plate was fit in three patients, while the 8 holes was fit for six patients. Whereas the 10-hole plate was fit in three patients. This proves that, a wide exposure can be achieved with the intraoral approach to accommodate even a 10 hole standard mini orbital plate which is comparable to all other skin approaches.

Upon our knowledge there is no available articles in literature that evaluated the infraorbital nerve affection using intraoral approach for management of infraorbital rim, there are several articles that reported infraorbital nerve affection.

Clinical evaluation of facial edema by using 3-lines measurements revealed that there were no significant facial asymmetry or disfigurement resulted after one month while it was highly obvious in the first week when comparing result to evaluation.

Other studies presented moderate surgical edema postoperatively and disappeared by the third to fifth postoperative day, presented facial edema in the current approach is due to the tissue manipulation and dissection to reach the infraorbital rim. However, the postoperative edema may be accepted to the patients rather than scaring or multiple incisions. However the main obstacle of the ability to drill in perpendicular direction which can be easily performed in the 6 holes. On the other hand, it has limited for accessing the orbital floor.

As for facial asymmetry $91.7 \%$ of patients noticed asymmetry before surgical procedure and all of them recorded improve of symmetry after the surgical procedure.

$50 \%$ of the patients recorded absence of pain sensation and no one of them registered severe pain but $41.7 \%$ said that the pain was mild, $8.3 \%$ moderate. Peisker et al stated 
that the duration of surgery seems to have a significant influence on satisfaction with pain intensity, desire for more pain medication, and vomiting ${ }^{[13]}$.

Patient satisfaction questionnaire included pain, numbness and facial asymmetry is $(91.7 \%)$ satisfied and (8.3\%) unsatisfied according to Q7, which achieve patient satisfaction.

\section{CONCLUSION}

Based on our results, we conclude that the intraoral approach is a valid technique for reduction and fixation of infraorbital rim that permits enough exposure and achieve patient satisfaction. Despite of increased edema, which spontaneously resolved within one month.

\section{CONFLICT OF INTEREST}

The authors declare no conflict of interest.

\section{REFERENCES}

1. Gomes de Souza Carvalho AC, Pereira CC, Queiroz TP and Magro-Filho O. Intraoral Approach to Zygomatic Fracture: Modified Technique for Infraorbital Rim Fixation. J Craniofac Surg 2012; 23: 537 - 538 .

2. Yonehara Y, Hirabayashi S, Tachi M, et al. Treatment of zygomatic fracture without inferior orbital rim fixation. J Craniofac Surg 2005; 16: $481 \mathrm{Y} 485$.

3. Zachariades N, Mezitis M, Anagnostopoulos D (1998) Changing Trends in the treatment of zygomaticomaxillary complex fractures: a 12-year evaluation of methods used. J Oral Maxillofac Surg 56 (10): $1152-1156$.

4. Fonseca. Oral and Maxillofacial surgery (2000) Trauma-Vol. 3, 1st Edition, 159 - 163.

5. El-Anwar MW, Elsheikh E, Sweed AH, Ezzeldin $\mathrm{N}$ (2015) Electromyography assessment in zygomaticomaxillary complex fractures. Oral and Maxillofacial Surgery 19 (4): 375 - 379.

6. Subramanian B, Krishnamurthy S, Kumar PS, Saravanan B and Padhmanabhan M. Comparison of various approaches for exposure of infraorbital rim fractures of zygoma. J Maxillofac Oral Surg 2009; 8 (2): $99-102$.

7. D. M. Laskin, Cirug'ra Bucal y Maxilofacial. Buenos Aires, Editorial Medica Panamericana S. A., 1987.

8. Hammuda A. Intraoral Approach for Reduction and Fixation of Infraorbital Rim Fracture. Egy J Oral Maxillofac 2018; 9 (4): 179 - 84.

9. Padmanavam A, Mishra S. Patient perspective in the management of zygomatic fractures. Ann Maxillofac Surg 2018; 8: 239 - 46.

10. de Souza Carvalho, Abrahao Cavalcante Gomes, et al. "Intraoral approach to zygomatic fracture: modified technique for infraorbital rim fixation." Journal of Craniofacial Surgery 23.2 (2012): 537 - 538.

11. Shikara, Meryam A., Kalpesh T. Vakharia, and Jewel D. Greywoode. "Zygomaticomaxillary Complex-Orbit Fracture Alignment: Comparison of Buccal and Orbital Approach Techniques." JAMA facial plastic surgery 20.5 (2018): 428 - 429.

12. Yonehara Y, Hirabayashi SH, Tachi M, Ishii H. Treatment of zygomatic fractures without inferior orbital rim fixation Journal of Craniofacial Surgery 2005, 16 (3): 481 - 5.

13. Kurita M, Okazaki M, Ozaki M, et al. Patient satisfaction after open reduction and internal fixation of zygomatic bone fractures. Journal of Craniofacial Surgery 2010; 21 (1): 45. 